\title{
Transhepatic intraductal radiofrequency ablation of a biliary papillary mucinous neoplasm
}

Recently, biliary tract intraductal papillary mucinous neoplasm (IPMN) was described as a rare but important preinvasive lesion coinciding with pancreatic IPMN [1]. Several studies have revealed dysregulated molecular pathways involving mutations of the GNAS and KRAS genes and have tried to distinguish between biliary IPMN and pancreatic IPMN by means of their mucin-subtype pattern [2]. Nevertheless, the majority of data on biliary tract IPMNs come from retrospective studies, mostly where surgical interventions were performed [3]. Only one case report has so far described a combined chemoradiotherapy approach in a patient with synchronous biliary tract and pancreatic IPMNs [4].

Here we present the complex case of a patient with a biliary tract IPMN that, after initial surgery, was successfully treated by intraductal transhepatic radiofrequency ablation (RFA) via a transcutaneous access route ( $\vee$ Video 1$)$. The 71-year-old man had a diagnosis of arterial hypertension and type 2 diabetes mellitus. The patient was transferred from a primary care hospital. Initially, he underwent a laparoscopic cholecystectomy because of cholecystitis. A revision of the bile duct with T-drain implantation and subhepatic drains was necessary because of postsurgical bile duct leakage. A computed tomography (CT) scan was suspicious for an intrahepatic tumor but was not reproducible. Initially, we visualized the bile duct via the T-drain and found contrast defects that were felt likely to be bile duct stones. However, endoscopic retrograde cholangiopancreatography (ERCP) found no calculi, although viscous mucus was removed by means of a balloon and a basket catheter. Interestingly, magnetic resonance cholangiopancreatography (MRCP) revealed an inconspicuous bile duct.

In the following weeks, the patient suffered from relapsing cholangitis due to episodes of mucus-induced bile duct

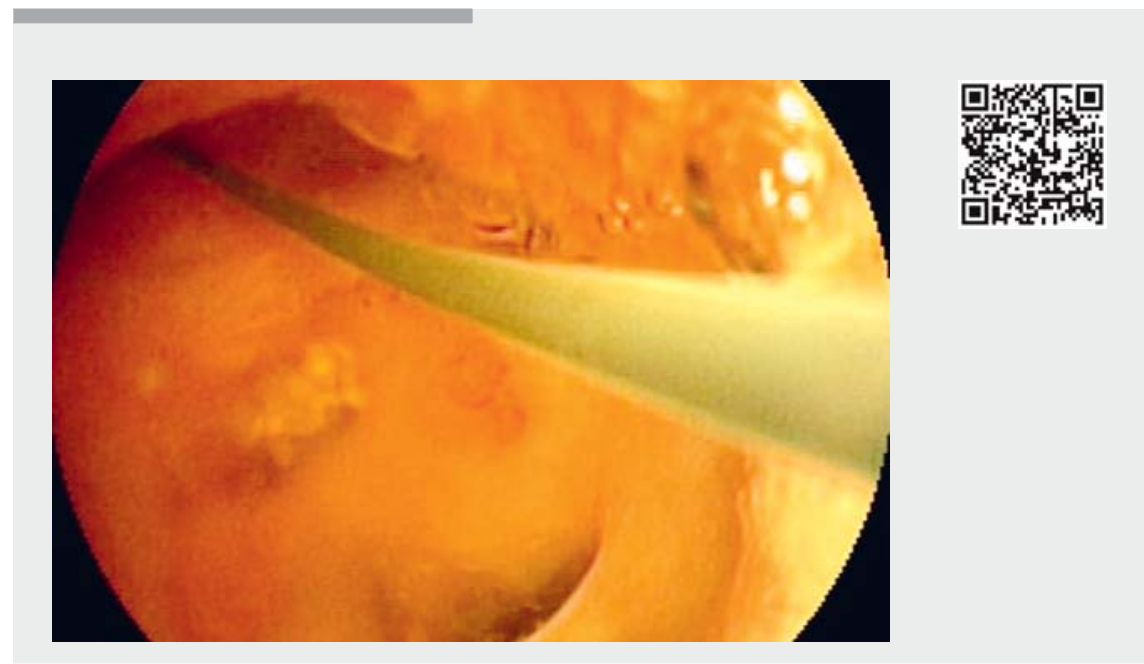

$\checkmark$ Video 1 The course and procedures performed for a patient with biliary duct intraductal papillary mucinous neoplasm (IPMN). After undergoing an initial hemihepatectomy, the patient suffered from recurrent mucin production and cholangitis. Transhepatic cholangioscopy showed a biliary IPMN in the remaining bile duct, which was treated by intraductal radiofrequency ablation (RFA).

stent occlusion. Mother-baby cholangioscopy was infeasible because the viscous mucus could not be removed. Finally, ERCP showed a stenosis of the right intrahepatic bile duct that revealed signal enhancement on a positron emission tomography-CT (PET-CT) scan.

Following an interdisciplinary meeting, we recommended to the patient that he undergo a right-sided hemihepatectomy for a tentative diagnosis of a mucin-producing tumor, and this was subsequently performed without difficulty. However, the histology showed a low grade biliary IPMN with an $\mathrm{R} 1$ resection.

In the next few months, the patient suffered from recurrent bile duct stent occlusions, cholangitis, and bilioma. We therefore decided, owing to unsatisfactory ERCP treatment, to perform left-sided percutaneous transhepatic cholangiodrainage (PTCD). Over a period of 6 weeks, this transhepatic access to the bile duct was dilated to 18-Fr. Thus, we created a large enough approach tract to be able to insert a small diameter endoscope and conduct a wire-guided transhepatic cholangioscopy. Through this, we were able to observe and confirm the presence of a biliary IPMN in the remaining bile duct. In addition, transhepatic intraductal ablation of the biliary IPMN was successfully performed by RFA using the Habib EndoHPB ablation catheter, with no immediate or delayed complications. Following the RFA treatment, the patient has shown no signs of cholangitis or recurrent biliary IPMN during 1 year of follow-up.

To the best of our knowledge, this is the first report of transhepatic intraductal RFA of a biliary duct IPMN performed via transcutaneous access. However, randomized controlled trials will be necessary to further assess this interventional technique.

Endoscopy_UCTN_Code_TTT_1AR_2AF 


\section{Competing interests}

None

The authors

Marcus Hollenbach ${ }^{1}$, Jürgen Feisthammel ${ }^{1}$, Joachim Mössner ${ }^{1}$, Tobias Schlosser ${ }^{1}$, Thomas Kahn², Osama Sabri' ${ }^{3}$, Albrecht Hoffmeister ${ }^{1}$

1 Department of Medicine, Neurology and Dermatology, Division of Gastroenterology and Rheumatology, University of Leipzig, Leipzig, Germany

2 Department of Diagnostic and Interventional Radiology, University of Leipzig, Leipzig, Germany

3 Department of Nuclear Medicine, University of Leipzig, Leipzig, Germany

Corresponding author

\section{Marcus Hollenbach, MD}

Department of Medicine, Neurology and Dermatology, Division of Gastroenterology and Rheumatology, University of Leipzig, Liebigstrasse 20, D-04103 Leipzig, Germany marcus.hollenbach@medizin.uni-leipzig.de

\section{References}

[1] Rocha FG, Lee H, Katabi $\mathrm{N}$ et al. Intraductal papillary neoplasm of the bile duct: a biliary equivalent to intraductal papillary mucinous neoplasm of the pancreas? Hepatology 2012; 56: $1352-1360$

[2] Fukumura Y, Nakanuma $Y$, Kakuda $Y$ et al. Clinicopathological features of intraductal papillary neoplasms of the bile duct: a comparison with intraductal papillary mucinous neoplasm of the pancreas with reference to subtypes. Virchows Arch 2017; 471: 65-76

[3] Wang X, Cai YQ, Chen YH et al. Biliary tract intraductal papillary mucinous neoplasm: report of 19 cases. World J Gastroenterol 2015; 21: $4261-4267$

[4] Valente R, Capurso G, Pierantognetti P et al. Simultaneous intraductal papillary neoplasms of the bile duct and pancreas treated with chemoradiotherapy. World J Gastrointest Oncol 2012; 4: 22-25

\section{Bibliography}

DOI https://doi.org/10.1055/a-0889-7116

Published online: 9.5.2019

Endoscopy 2019; 51: 897-898

(c) Georg Thieme Verlag KG

Stuttgart · New York

ISSN 0013-726X
ENDOSCOPY E-VIDEOS

https://eref.thieme.de/e-videos

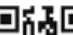

Endoscopy E-Videos is a free

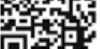
access online section, reporting on interesting cases and new techniques in gastroenterological endoscopy. All papers include a high quality video and all contributions are freely accessible online.

This section has its own submission website at https://mc.manuscriptcentral.com/e-videos 